\title{
Near-field Signals Detected by a Standard Bipolar Electrode without Detection of Corresponding Signals by Microelectrode: What is the Mechanism?
}

\author{
Takashi Nakashima ${ }^{1}$, Cyril Goujeau ${ }^{2}$, Yosuke Nakatani ${ }^{3}$, Ghassen Cheniti ${ }^{4}$, Pierre Jais $^{5}$, \\ and Frederic Sacher ${ }^{6}$ \\ ${ }^{1}$ Hôpital Cardiologique du Haut-Lévêque-CHU de Bordeaux, Université de Bordeaux \\ ${ }^{2}$ Hôpital Cardiologique du Haut Lévêque, Service de rythmologie-électrophysiologie et \\ stimulation cardiaque \\ ${ }^{3}$ Hôpital Cardiologique du Haut-Lévêque-CHU de Bordeaux, Université de Bordeaux \\ ${ }^{4}$ Hôpital Cardiologique du Haut Lévêque \\ ${ }^{5}$ Centre Hospitalier Universitaire de Bordeaux \\ ${ }^{6}$ Bordeaux University Hospital
}

April 27, 2020

Near-field Signals Detected by a Standard Bipolar Electrode without Detection of Corresponding Signals by Microelectrode: What is the Mechanism?

Short title ; Near-filed Microelectrode signal

Takashi Nakashima, MD*, Cyril Goujeau, MD, Yosuke Nakatani, MD,

Ghassen Cheniti, MD, Pierre Jaïs, MD, Frédéric Sacher, MD

All authors have no conflicts of interest to disclose.

Department of Cardiac Electrophysiology, Service de Rhythmologie, Hôpital Cardiologique du Haut-Lévêque (Centre Hospitalier Universtaire de Bordeaux)

Avenue de Magellan, 33604, Bordeaux-Pessac, France

Correspondence to Takashi Nakashima, MD

Department of Cardiac Electrophysiology, Service de Rhythmologie, Hôpital Cardiologique du Haut-Lévêque (Centre Hospitalier Universtaire de Bordeaux)

Avenue de Magellan, 33604, Bordeaux-Pessac, France

Tel: +33557656863 19, Fax: +33557656896 20, Email; takashin727jw@yahoo.co.jp, ORCID: 00000002-8538-9259

Key words: Ablation; Electrogram analysis; Microelectrode; Ventricular tachycardia

\section{Case report}

A 77-year-old woman with ischemic cardiomyopathy was referred for radiofrequency catheter ablation (RFCA) for recurrent ventricular tachycardia (VT). Mapping was performed during atrial fibrillation paced at 60 per minute by the implanted cardioverter defibrillator because of hemodynamically unstable VT. RFCA 
was performed by targeting local abnormal ventricular activity (LAVA $)^{1}$ using a 3.5 -mm-tip open-irrigated catheter equipped with three microelectrodes (MEs: Qdot Micro ${ }^{\text {TM}}$; Biosense Webster, Diamond Bar, California). While mapping the left ventricle, the standard bipolar electrode detected LAVA, whereas the MEs did not detect the corresponding signals (Figure 1). What is the mechanism underlying these apparently paradoxical electrograms?

\section{Commentary}

The MEs are circumferentially located on the distal tip of the standard electrode (Figure 2A). ${ }^{2}$ This configuration is different from that of the $\mathrm{MiFi}^{\mathrm{TM}}$ IntellaTip ablation catheter, in which MEs are located in a radial fashion on its lateral aspects (Boston Scientific, Natick, MA). ${ }^{3}$ Therefore, when the distal end of the electrode equipped with MEs is in contact with the tissue perpendicularly, the MEs detect the signals (Figure 2B). However, when the catheter is in contact to the tissue parallelly, there is poor contact between the MEs and tissue; therefore, the standard bipolar electrode detects the signals and the MEs do not (Figure $2 \mathrm{C})$.

Because of their size and interelectrode spacing, MEs can detect near-field signals that the standard bipolar electrode fails to detect. Therefore, MEs improve identification of local electrical activity responsible for arrhythmias. ${ }^{2-5}$ Bipolar electrograms depend on the contact between the tissue and electrode for recording electrical signals, and on the orientation of the catheter relative to the tissue (from parallel to perpendicular). ${ }^{2-5}$ The present case described the use of both the standard bipolar electrode and MEs for simultaneously recording the local electrical activity (i.e., LAVA), wherein the standard bipolar electrode recorded LAVA without any corresponding signals being detected on the MEs. This phenomenon may be attributed to the orientation of the catheter relative to the tissue and the configuration of the MEs.

\section{References}

1. Jaïs P, Maury P, Khairy P, Sacher F. Nault I. Komatsu Y. Hocini M. Forclaz A. Jadidi A. S. Weerasooryia R. Shah A. Derval N. Cochet H. Knecht S. Miyazaki S. Linton N. Rivard L. Wright M. Wilton S. B. Scherr D. Pascale P. Roten L. Pederson M. Bordachar P. Laurent F. Kim S. J. Ritter P. Clementy J. Haissaguerre M. Elimination of local abnormal ventricular activities: a new end point for substrate modification in patients with scar-related ventricular tachycardia. Circulation . 2012;125:2184-2196.

2. Lee A, Walters TE, Alhede C, Vittinghoff E, Sievers R, Gerstenfeld EP. Standard peak-to-peak bipolar voltage amplitude criteria underestimate myocardial scar during substrate mapping with a novel microelectrode catheter. Heart Rhythm . 2019;S1547-5271(19)30919-1.

3. Lloyd MS, Hoskins MH, Shah AD, Langberg JJ. Electrogram Characteristics of Ablated and Non-Ablated Myocardium in Humans: A Comparison of Miniaturized Embedded Electrodes and Conventional Ablation Electrodes. J Cardiovasc Electrophysiol . 2016;27:820-824.

4. Leshem E, Tschabrunn CM, Jang J, Whitaker J. Zilberman I. Beeckler C. Govari A. Kautzner J. Peichl P. Nezafat R. Anter E. High-Resolution Mapping of Ventricular Scar: Evaluation of a Novel Integrated Multielectrode Mapping and Ablation Catheter. JACC Clin Electrophysiol . 2017;3:220-231.

5. Tschabrunn CM, Roujol S, Dorman NC, Nezafat R, Josephson ME, Anter E. High-Resolution Mapping of Ventricular Scar: Comparison Between Single and Multielectrode Catheters. Circ Arrhythm Electrophysiol . 2016;9:10.1161/CIRCEP.115.003841 e003841.

\section{Figure 1}




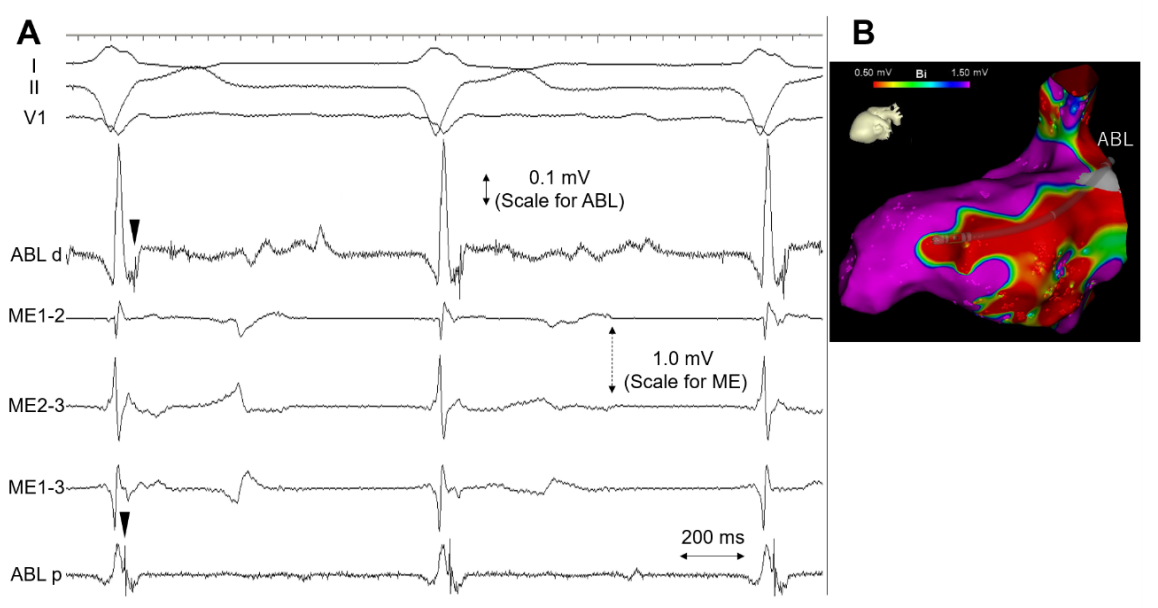

Figure 1 (A). Simultaneously recording electrograms using the standard bipolar electrodes and microelectrodes (MEs). The standard bipolar electrode detects near-field signals (arrows heads), whereas the MEs did not detect any near-field signals corresponding to these signals. Vertical solid and dotted arrows indicate the scale of $\mathrm{ABL}$ and $\mathrm{ME}$, respectively. ABL, ablation catheter; $\mathrm{ME}$, microelectrode; $\mathrm{d}$, distal; p, proximal. (B). The orientation of the catheter to the tissue on the three-dimensional mapping system.

\section{Figure 2}

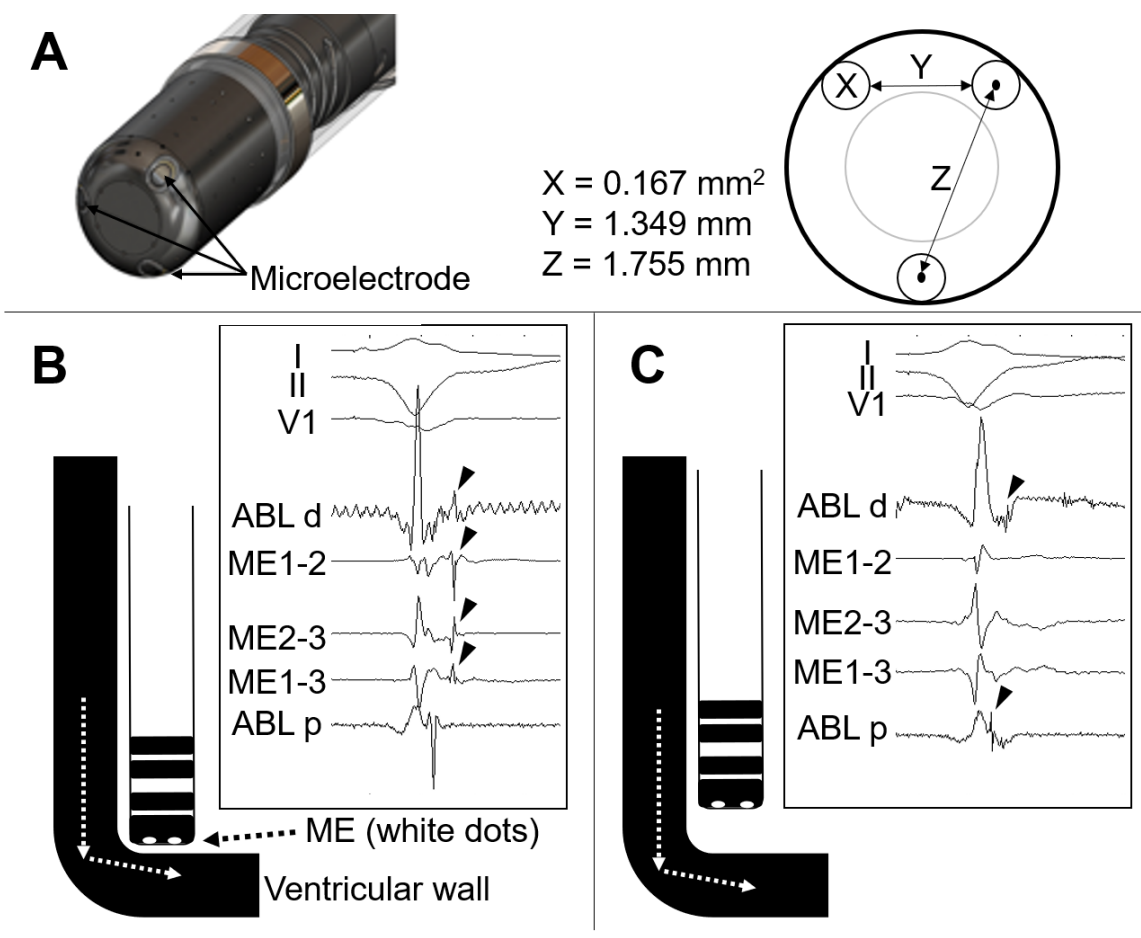

Figure 2 (A). Ablation catheter with three microelectrodes (MEs) circumferentially equipped on the distal tip (Qdot Micro ${ }^{\mathrm{TM}}$; Biosense Webster, Diamond Bar, California).

(B) The standard bipolar electrode and MEs in contact with the tissue. Note that the signals were detected by both the standard bipolar electrode and MEs (arrow heads). 
(C) The catheter in contact with the tissue parallelly, leading to a lack of contact between the MEs and the tissue. Note that the near-field signals were detected by the standard bipolar electrode (arrow heads) without detection of any corresponding signals by the ME. Abbreviations as in Figure 1. 